\title{
Classification of Phantom Finger, Hand, Wrist and Elbow Voluntary Gestures in Transhumeral Amputees with sEMG
}

\author{
N. Jarrassé, C. Nicol, A. Touillet, F. Richer, N. Martinet, J. Paysant and J.B. de Graaf.
}

\begin{abstract}
Decoding finger and hand movements from SEMG electrodes placed on the forearm of transradial amputees has been commonly studied by many research groups. A few recent studies have shown an interesting phenomenon: simple correlations between distal phantom finger, hand and wrist voluntary movements and muscle activity in the residual upper arm in transhumeral amputees, i.e., of muscle groups that, prior to amputation, had no physical effect on the concerned hand and wrist joints. In this study, we are going further into the exploration of this phenomenon by setting up an evaluation study of phantom finger, hand, wrist and elbow (if present) movement classification based on the analysis of surface electromyographic (sEMG) signals measured by multiple electrodes placed on the residual upper arm of 5 transhumeral amputees with a controllable phantom limb who did not undergo any reinnervation surgery. We showed that with a state-of-the-art classification architecture, it is possible to correctly classify phantom limb activity (up to 14 movements) with a rather important average success (over $80 \%$ if considering basic sets of 6 hand, wrist and elbow movements) and to use this pattern recognition output to give online control of a device (here a graphical interface) to these transhumeral amputees. Beyond changing the way the phantom limb condition is apprehended by both patients and clinicians, such results could pave the road towards a new control approach for transhumeral amputated patients with a voluntary controllable phantom limb. This could ease and extend their control abilities of functional upper limb prosthetics with multiple active joints without undergoing muscular reinnervation surgery.
\end{abstract}

\section{CONTEXT}

An arm amputation is extremely invalidating since many of our daily tasks require bimanual and precise control of hand movements. Major arm amputations are mainly the consequence of accidents and pathology and therefore touch all social layers. In France in 2013, about 200 major arm amputations were performed of which $60 \%$ concerned the upper-arm (elbow disarticulation, trans-humeral amputation, scapula-humeral disarticulation). It is well established that a number of neuromuscular reorganisation phenomena occur after the amputation. Indeed, the cortical regions of which the neuronal activity was related to the control and sensory

N. Jarrassé and F. Richer are with the CNRS, UMR 7222, ISIR / INSERM, U1150 Agathe-ISIR, Sorbonne University, UPMC Univ Paris 06, Paris, France.

C. Nicol and J. de Graaf are with the Institute of Movement Sciences, UMR 7287 - CNRS \& Aix-Marseille University, Marseille, France.

A. Touillet, N. Martinet and J. Paysant are with the Institut Rgional de Radaptation, UGECAM Nord-est, Nancy France. information processing of the now missing limb, will gradually be active in relation to another, most often adjacent, part of the body [1], [2]. This reorganization often goes together with a vivid perception of the presence of the missing limb, called "phantom limb". The phantom limb can be the object of mechanical, thermal and, unfortunately, painful sensations (see [3] for a review). A lesser known phenomenon is that the phantom limb can often be moved at will [4], [5]. Although these voluntary movements of the phantom limb are slower and more effortful than those of an intact limb, the patients feel the movements to be made in correspondence with their will and they are able to imitate with their intact hand the movements they perform with their phantom hand [6]. Since the prevalence of mobile phantoms is missing in the literature we recently performed an epidemiological study in upper limb amputees. It appears that out of 30 arbitrarily chosen interviewed upperlimb amputees, 23 patients have a mobile phantom limb, i.e., $85 \%$ [7]. Moreover, out of 7 patients amputated more than 10 years ago, 5 describe persistent phantom movements, even 48 years after amputation. This prevalence of mobile upper limb phantoms is clearly higher than one could have expected given the lack of attention generally given to this phenomenon in the literature. Results of several studies suggest that, despite the cortical reorganization, the primary somatosensory cortex (P1) can still process somatosensory information from the missing limb [8] and, moreover, the primary motor cortex (M1) still can send motor commands to the missing limb [9], [10]. These motor commands, being unable to project on the missing limb muscles, project on the preserved muscles of the residual limb, resulting in muscle activation patterns which are specific to the type of executed phantom movement [9], [11]. Indeed, distinct phantom movements of the amputated body parts are accompanied by distinct sEMG patterns in the remaining proximal muscles that normally would not have been activated. Although this is not surprising for forearm amputees (whose finger muscles are located in the forearm and thus still present), it is important to remind that this phenomenon can be observed in upper-arm amputees where phantom hand and wrist movements are associated with muscle activity at the upper-arm and shoulder levels, i.e., in muscles groups that are normally not involved in hand and wrist movements. The goal of this study is to determine whether residual upper arm muscle activity patterns associated to phantom hand, wrist and elbow voluntary movements [9] can be identified using a noninvasive multi-electrode array and state-of-the-art classification (i.e., pattern recognition) algorithms, and that these patterns are 


\begin{tabular}{|c|c|c|c|c|c|c|c|c|c|}
\hline Participant & Sex & Age (years) & Elapsed Time (months) & Amput. side & Dominant Limb? & Amput. type & Amput. cause & Pain treatment & Prosthesis \\
\hline P1 (S3) & $\mathrm{M}$ & 53 & 392 months & left & no & $1 / 3$ of the arm left & traumatic & no & Aesthetic (passive) or no prosthesis \\
\hline P2 (S4) & $\bar{F}$ & 77 & 165 months & right & yes & $1 / 3$ of the arm left & traumatic & no & Myoelectric \\
\hline P3 (S7) & $\overline{\mathrm{M}}$ & 33 & 42 months & right & yes & $2 / 3$ of the arm left & traumatic & yes & Myoelectric or no prosthesis \\
\hline $\begin{array}{ll}\mathrm{P} 4 \text { (S9) } \\
\end{array}$ & $\mathrm{M}$ & 24 & 12 months & left & zes & $2 / 3$ of the arm left & traumatic & yes & Myoelectric \\
\hline P5 (S17) & $\bar{M}$ & 62 & 67 months & left & no & $1 / 3$ of the arm left & traumatic & no & Myoelectric \\
\hline
\end{tabular}

TABLE I.

DEMOGRAPHIC DATA CONCERNING THE FIVE PARTICIPANTS. M=MALE; F=FEMALE; ELAPSED TIME = TIME SINCE AMPUTATION

"PROSTHESIS" INDICATES THE TYPE OF PROSTHESIS THE PATIENTS USUALLY WEARS.

strong, robust and controllable enough to be used to perform a real-time control task. If this is the case, for patients having phantom movements (i.e., over $80 \%$ [7]), a surgical method such as Targeted Muscle Reinnervation (TMR) [12] could possibly be not always necessary in order to gain enough signals to control a high-degrees-of-freedom prosthesis of the hand and arm.

\section{METHODS}

We present in this paper the results of two distinct experimental sessions: one simple recording session (to acquire data to perform preliminary offline classification tests on the recorded signals) to first estimate the possibility of identifying these patterns associated to phantom limb, and then one more realistic control task during which the patients had to mobilize their phantom limb to activate associated buttons on a graphical user interface.

\section{A. Participants}

Five patients with a unilateral transhumeral amputation were selected to participate to the study ( 4 men and 1 woman, aged 33 to 77 years). The selection of our participants was based on the level of amputation (only transhumeral amputees), their control ability of a mobile phantom limb (i.e., the possibility to perform several different phantom movements), the absence of phantom limb pain, and on the availability of the patient during the period of recording. The patients are followed-up regularly at the Louis Pierquin Centre of the Regional Institute of Rehabilitation, Nancy, France. For all participants, the amputation was of traumatic origin. Table I resumes demographic data. All of them provided written informed consent to participate in the study along with permission for publication of photographs for scientific and educational purposes. The protocol was approved by the Local Ethical Committee of the Institut Régional de Readaptation (IRR) and performed in accordance with the Declaration of Helsinki.

Their voluntary mobilization of phantom limb was discovered through a questionnaire and a preliminary evaluation in order to make clear distinctions between sensations in the residual limb, phantom pain, phantom sensations, and most importantly, between mobility of the residual limb and that of the phantom limb [7]. Table II presents the list of the phantom movements that each participant was able to perform. All five participants reported a good feeling and control of their phantom hand, including individual control of some fingers, of wrist rotations (except for participant P3) and, for three of them, of their phantom elbow (flexion and extension). It is important to underline that movement possibilities varied a lot from one participant to another: for example P2 could

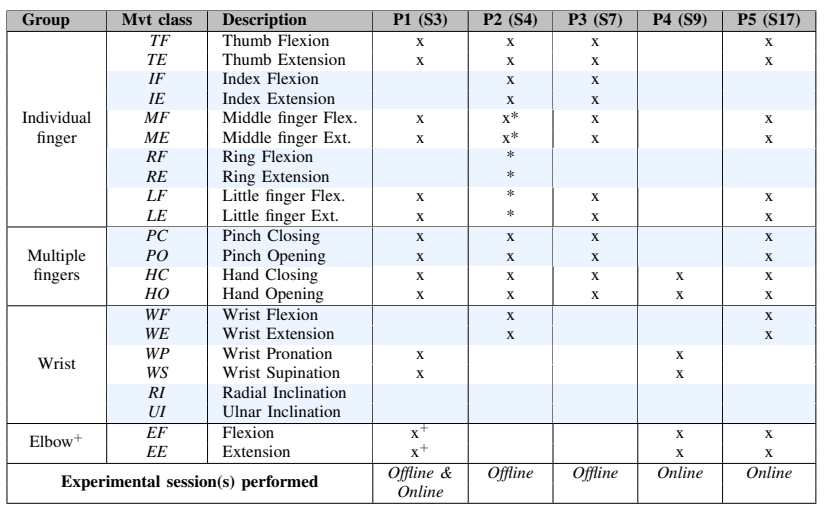

TABLE II. PERFORMED PHANTOM LIMB MOVEMENTS FOR EACH PARTICIPANT (* INDICATES THAT THE MOVEMENTS $M F, R F, L F$ AND $M E$, $R E, L E$ WERE COUPLED TOGETHER; ${ }^{+}$INDICATES THAT ELBOW MOVEMENTS WERE ONLY PERFORMED DURING THE ONLINE CONTROL SESSION).

not individually control the middle, ring and little fingers, P1 could not voluntarily control his index finger (like P3) and his ring finger (similarly to all participants) and P4 was only able to control his whole hand and had no individual control over his fingers. Apart from P4, all participants were at least able to perform voluntarily movements of thumb, major and little fingers along with pinch and whole hand opening and closing actions, and at least one wrist movements. Since the two sessions dit not occur on the same day, only one patient could participate to both sessions. Therefore, the other two patients in each session only participated in either the first or the second session.

\section{B. Protocols}

1) Offline classification of pre-recorded repetitive sequences: For three participants (P1,P2 and P3, see Table II), a recording session was performed during which surface EMG (sEMG) activity of the residual limb was recorded while the participants executed movements of the phantom hand and wrist (if present), and synchronously imitated the phantom limb movement with their intact and instrumented hand (see Fig.1).

Based on recording of the kinematics of the intact hand mimicking the phantom hand action, the sEMG data from the residual limb (see below for the description of the experimental setup that was used) was cut and labeled with the corresponding phantom limb movement (motion class). In order to assess the performance of the classification algorithm, half of the recorded data (sEMG tagged with motion class 


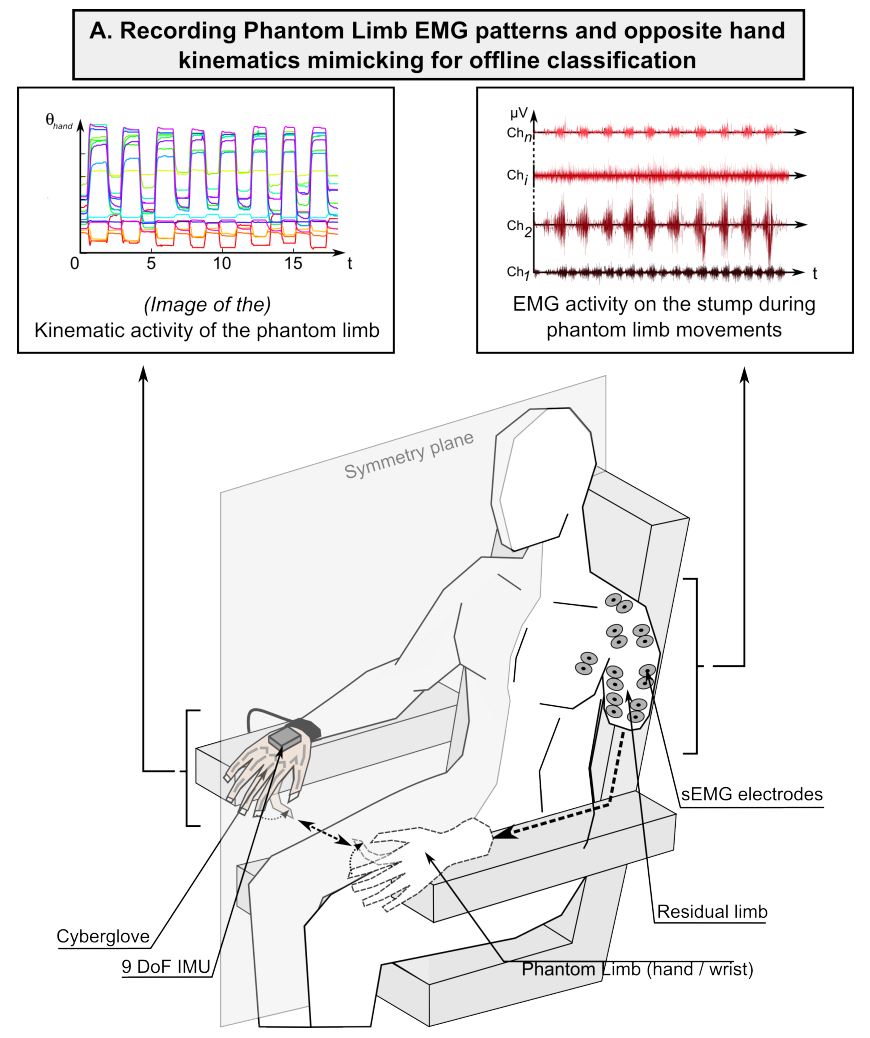

Fig. 1. Global view of the experimental setup during recording session for offline classification. Examples of kinematic measurements recorded by the gloves and associated sEMG activity of participant P3 are shown.

labels) was used for the training phase, while the other half was cut into parts and mixed to create random sequences of sEMG activation patterns associated to known phantom limb movements and used during the testing phase. The performance of the classification algorithm is expressed as the gesture-recognition success rate. The effort required for execution of each phantom movement determined the order of the tested movement sequence, from the easiest to the hardest one. Each type of phantom movement was tested in a sequence lasting maximally $40 \mathrm{~s}$ of cyclic repetitions at a comfortable frequency (no velocity instruction was given to the participants). The patient comfortably seated in a specific dedicated chair, fitted with armrests and a head rest, was instructed to synchronously mimic the phantom movements with the intact limb. Each sequence was followed by a resting period of several minutes. The experimenter verbally indicated the beginning and end of each period. If after some cyclic movements the patient could not move the phantom limb anymore (generally caused by fatigue) or, on the contrary, the patient had performed 10-15 cycles before the end of the $40 \mathrm{~s}$ period, the recording was stopped. At the end of each sequence, the patient reported the experienced difficulty of the phantom movement execution and the quality of the mimicking with the intact hand. When the patient estimated that the mimicking was not correctly done, the sequence was repeated if possible for the patient. If not, the sequence was not taken into account for data analysis. Once the participant had performed 10 to 15 repetitions of the phantom limb actions listed in Table II (mimicked in real-time by the intact limb), sEMG recordings were split into two parts (training and testing parts). For each sEMG recording, the data from the associated kinematic recording (Cyberglove or IMU) of the intact hand mimicking the phantom limb were used to automatically cut and label the sEMG recordings with their associated movement class thanks a dedicated Matlab ${ }^{\odot}$ script. The training parts of the labeled sEMG files were then used to train the classifier while the testing parts of the labeled sEMG files were assembled into a random sequence (different for each participant) and sent to the offline classifier to evaluate its performance.

2) Online control of a graphical user interface: Three participants (P1, P4 and P5) were recruited for this experiment. They were again comfortably seated in a specific dedicated chair, fitted with armrests and a head rest, but this time a screen showing the online control graphical user-interface was placed in front of the participant (see Fig. 2. Once the electrodes were

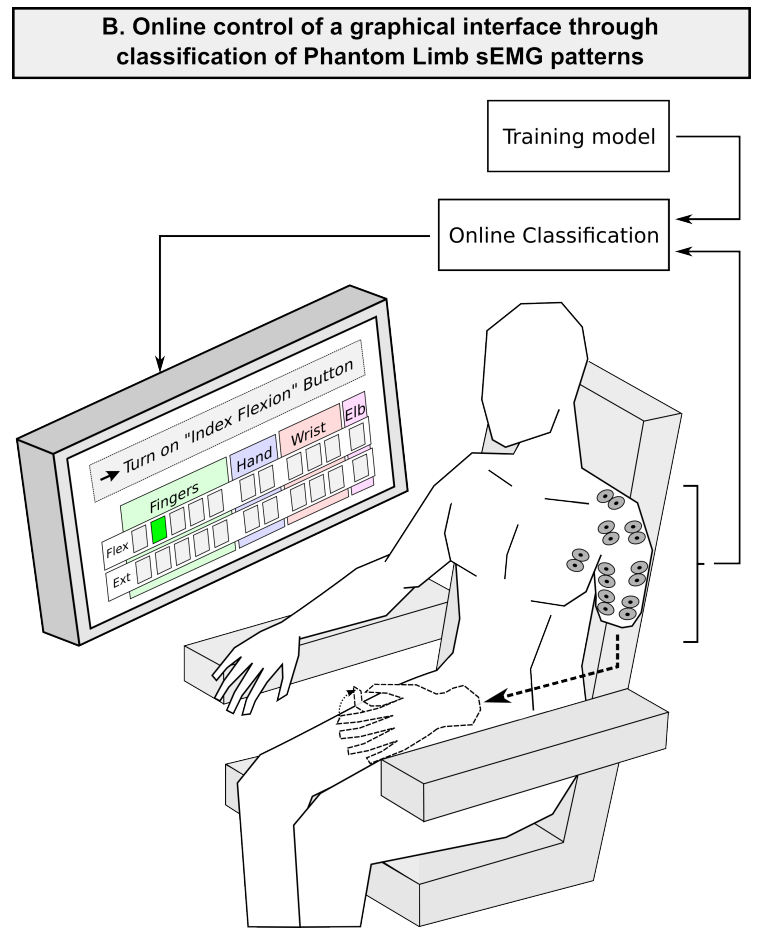

Fig. 2. Global view of the experimental setup for online control of a graphical user interface through the mobilization of the mobile phantom limb and the real-time classification of associated sEMG pattern on the residual limb. Here an example for flexing of the index is shown.

placed, the session started with a training phase during which the sEMG activities of the residual limb were recorded. The three participants were asked to successively perform once all possible phantom movement with a few seconds of rest inbetween each two movements. This sequence was repeated once ( 2 demonstrations per movement). The experimenter was in charge of verbally asking the subject to execute a given 


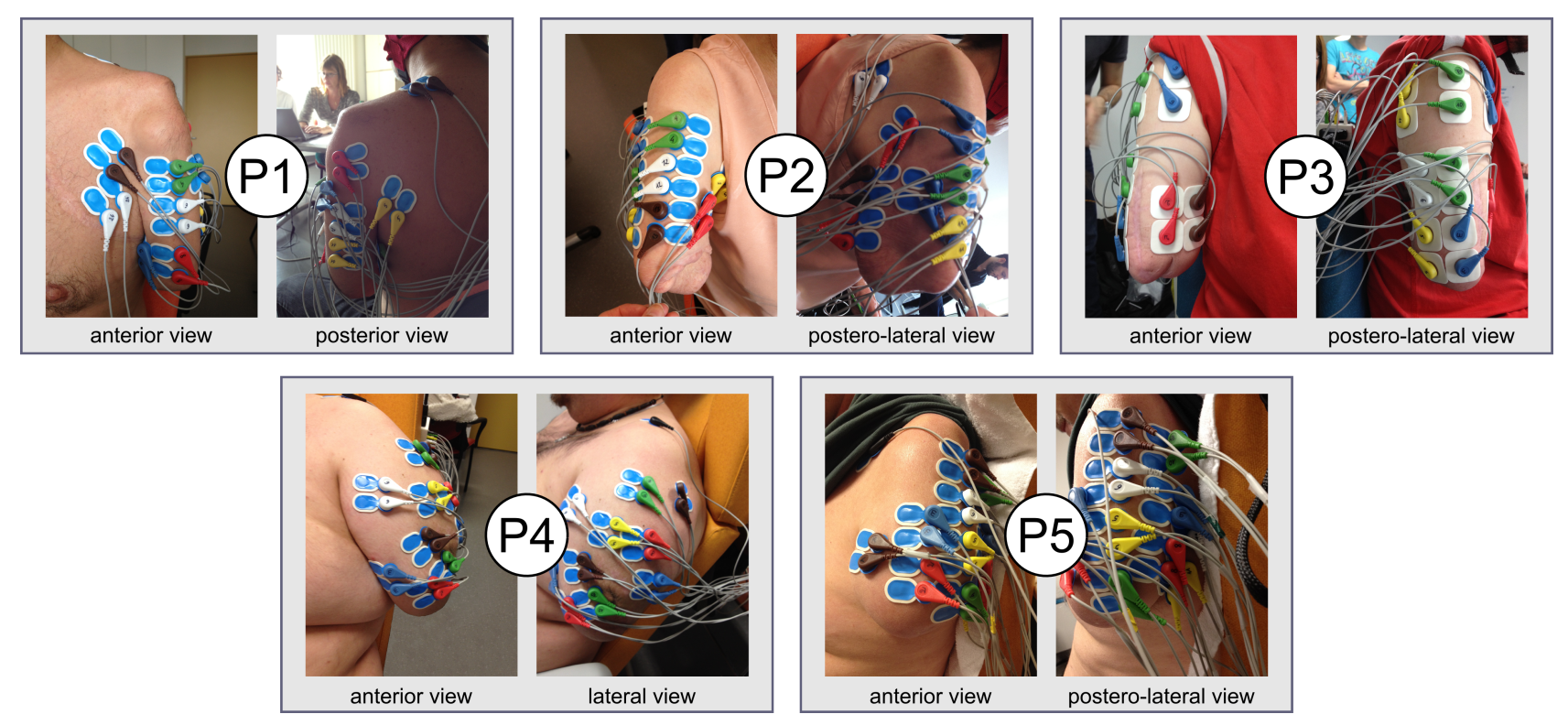

Fig. 3. Electrode placements on all five participant's residual limb.

movement and therefore was in charge of the rhythm of the performance. No instruction was given about the amplitude and the velocity of the gesture. Then, the recordings were manually tagged offline with the movement type (from Thumb Flexion $\mathrm{TF}$ to Elbow Extension EE) by the experimenter through a dedicated interface. This lasted no more than 5 minutes. Once the tagging was performed, the data were analyzed and assembled to constitute the training set of data to be used in the testing phase (online classification of the phantom limb activity and real-time control of the graphical user interface).

During the testing phase, while the online classification algorithm was running, the participants were asked to switch on specific buttons of the graphical user interface by performing specific phantom limb movements, each button being associated to a specific phantom movement. They were instructed to maintain the button switched on for two seconds by holding their phantom limb action during the whole time (since no latch algorithm was used) with a minimal amount of flickering (none if possible). The experimenter gave the instruction and then let the participant perform the task for 5 seconds. During this time, the participant had to try to maintain the desired button switched on for two seconds once, but could try another time if he couldnt perform it at his first attempt. Once the participant had performed the action (considered to be done even with little flickering) or if the 5 seconds had passed, the experimenter asked the subject to relax, and then moved on to the next action.

A list of pseudo-randomized movements (in order to avoid incompatible successions of actions like a finger extension after a hand opening) of their phantom limb (5 repetitions of each possible movements), all interspersed with pauses (usually a few minutes of rest between groups of 10-20 movements), was tested this way.

\section{Experimental setup}

1) Measurement of the surface myoelectric activity of the residual limb: A dedicated electrophysiological signalrecording system (Eegosports from ANT-Neuro ${ }^{\circledR}$, The Netherland) with 24 bipolar channels at 24-bit resolution was used to record sEMG activity of participant's residual limb at a $1 \mathrm{kHz}$ frequency. Because of the variability in residual limb length and muscle anatomy due to the level of amputation, the scheme of electrodes placement had to be adapted for each participant (see Figure 3). 12 pairs of sEMG electrodes (Ag/AgCl snap bipolar electrodes with a 1.25 -cm-diameter circular contact) were used for each participant to measure activity on various parts of the residual biceps, triceps, deltoid and sometimes trapezoidal and grand pectoralis muscles. No specific skin preparation was used before placing the active electrodes over the residual limb. The recorded sEMG signals were then filtered with a $[10 \mathrm{~Hz} ; 400 \mathrm{~Hz}]$ third-order bandpass Butterworth filter.

2) Measurement of the kinematics of the intact hand and wrist for offline classification: For the first session (offline classification on recordings), one right and one left Cyberglove $^{\circledR}$ II (18 sensors gloves among which only 15 were used) were used to record the five fingers of the intact hand mimicking the phantom hand movements, with a precision below one degree, at a frequency of $100 \mathrm{~Hz}$. Such devices rely on the use of piezoelectric sensors which are sewed inside the elastic glove at each joint level. Bending of a sensor generates a variation of its output voltage, proportionally to the angular posture of the joint. Even if the flexible glove adapt to many hand sizes, it has to be calibrated for each participant in order to get representatives values independent of the participant's hand morphology. We, therefore, developed a simple and fast calibration method derived from the procedure introduced in [13] which relies on the classical use of key- 

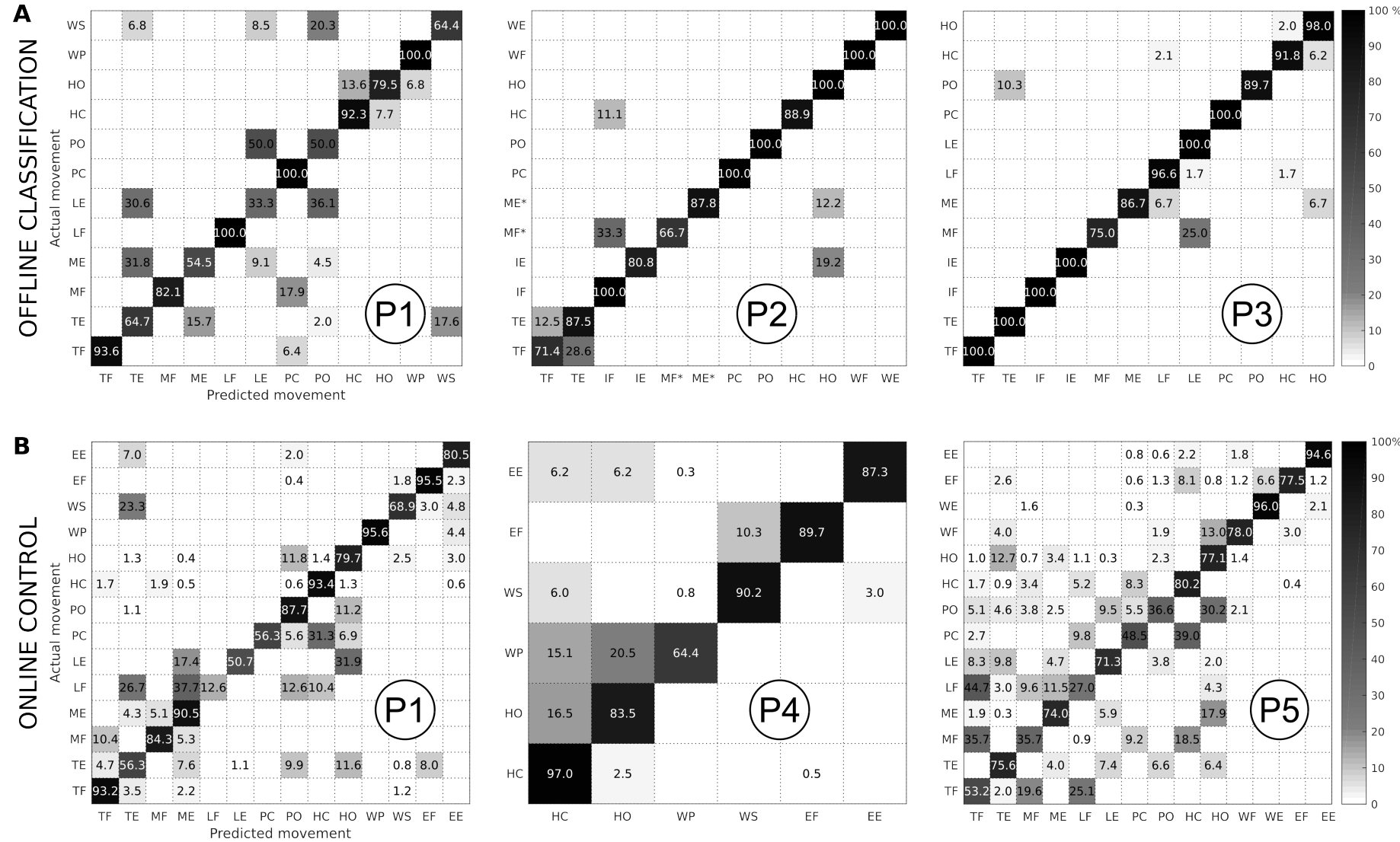

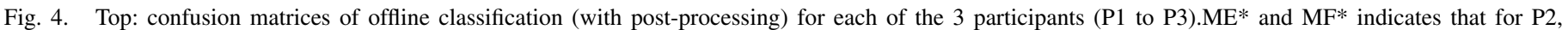

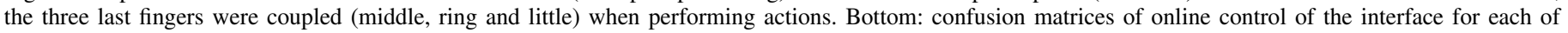

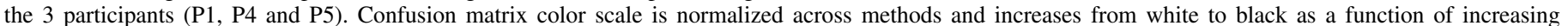
classification rate.

postures. A specific effort has been made to define these key-postures. During the calibration phase, each participant was wearing the glove and then asked to reproduce a certain number of known and defined postural patterns with their hand. These patterns are chosen in order to explore a large part of the angular displacement space of the human hand. As the theoretical angular values for this group of postures are known, a simple linear regression is then performed on the experimentally recorded values in order to identify the gain and offset values (for each sensor) to be applied to the raw data sent by the Cyberglove ${ }^{\odot}$ in order to get accurate angular measurements during the experiments. An optimal estimation through a least square minimization was performed over the set of recorded postures to identify these parameters. During our experiments, calibration was performed once by each participant before the recording session; the calibration data were kept and used during the entire session since the participants did not remove the glove during this time.

In order to track wrist movements, an additional Inertial Measurement Unit (IMU, 9 degrees-of-freedom Sensor Stick from Sparkfun $\left.{ }^{(}\right)$was attached to the top of the glove, allowing a precise reconstruction of the hand orientations in space (thanks to a dedicated sensor fusion algorithm calculating IMU orien- tations according to the accelerometer, gyroscope and magnetometer information) and of the range of the participant's wrist movements (flexion/extension, pronation/supination, ulnar/radial inclination). A dedicated interface was developed in $\mathrm{C}++$ relying on OpenGl and the use of multiple threads in order to ease the calibration phase for the experimenter and the patient (thanks to a real-time visualization function) and to perform precise synchronized $100 \mathrm{~Hz}$ acquisition of both the IMU and Cyberglove ${ }^{\odot}$ data. The recording of both the gloves and the IMU on one computer and the sEMG signals on another were started synchronously thanks to a dedicated SYNC pushbutton. The whole recording session was videotaped and lasted for about 45 minutes.

3) Real-time controllable Graphical User Interface: For the online control session, we used the BCI2000 software suite to develop a global control architecture on a desktop computer running Windows 7 (Intel Core i5-4690K (3.5 GHz) with 16 Go DDR3). BCI2000 is a general-purpose software suite designed for brain-computer interface (BCI) and was used here to run in parallel three principal modules: one acquisition driver to acquire the sEMG data (at a frequency of $1 \mathrm{kHz}$ ), one Matlab classification algorithm script (executed every $128 \mathrm{~ms}$ ), and one graphical user interface $(\mathrm{C}++$ with $\mathrm{Qt})$. This interface, 
which was presented to the participant during the task on a separate dedicated screen, consists in a wide window with four panels: one for fingers movements (flexion and extension of each finger), one for hand postures (whole hand and pinch closing and opening), one for wrist movements (three rotations in both directions), and finally one for elbow movements (flexion, extension). Within each panel, there is a large button ( $3 \mathrm{~cm}$ square) for each phantom movement (10 for the fingers panel, 4 for the hand panel, 6 for the wrist and 2 for the elbow ones). The button are directly controlled by the output of the classification algorithm: every $128 \mathrm{~ms}$, the movement class detected by the classifier was sent to the interface, if the associated confidence level is over a predefined ratio of $90 \%$, the button associated to the movement class turns from grey to light green.

4) Classification methods: The main goal of this study was to evaluate the possibility to recognize with state-of-the-art algorithms the muscle activity of the residual limb associated to the execution of phantom movements. The different aspects of the state-of-the-art classification architecture that was used are described below.

a) Classifier: In this study, we used a linear discriminant analysis (LDA) classifier [14] running on Matlab ${ }^{\odot}$. Many other classifications methods have been developed within the last decades (Multi-Layer Perceptrons (MLP) [15], Gaussian Mixture Models (GMM) [16] or fuzzy systems [17]), but we chose the LDA classifier for several reasons: it is based on a simple statistical approach and therefore it is computationally efficient (no "time costly" iterative training), it is robust [18] and does not require any parameter adjustment, while its classification performances are similar to more complex algorithms. [19].

b) Features: Features were computed from the sEMG using a 512-ms-sliding analysis window with a 128-ms-overlap between successive windows. Among the wide variety of features that have been investigated in the literature [18], we selected those known to be the most efficient and robust for classification of sEMG with LDA: the root mean square (RMS) value [20], the first 4 autoregressive coefficients (AR) ([21], [22]), the waveform length (i.e., the cumulative length of the waveform over the time segment) and the sample entropy [23] of the sEMG were extracted for each channel and used to create the feature vector. No dimensionality reduction was used.

c) Post-processing for offline classifcation: In order to remove transitional data, only for the offline classification of pre-recorded sequences, a smoothing operation was used on the classifier output. For this, analysis windows restricted to 512 ms before and after the movement transition were considered.

\section{Metrics}

1) Offline classification: the offline classification performance was calculated with Matlab $^{\odot}$ : similarly to the training data treatment, the testing sEMG recordings were automatically cut and labelled with their associated movement class thanks to a dedicated Matlab $^{\odot}$ script analyzing the associated kinematic recording of the intact hand mimicking the phantom limb. Confusion matrices were then generated by computing time ratios between movement class reference (given by the intact hand) and the output of the classifier.

2) Online experiment: the online performance was analyzed by a Matlab ${ }^{\odot}$ script to automatically determine within the 5 seconds interval allocated to each task (i.e., switching on one specific button), the start of the participant action (time instant when the classifier start detecting an action different from the inactivity with a confidence over 90\%) and its end (last time instant of the classifier output detection of an activity until the end of the $5 \mathrm{~s}$ time period). The confusion percentages were then calculated over these previously selected times of action, based on the ratio between time of action and time during which the correct button was turned on.

\section{RESULTS}

\section{A. Offline classification of phantom movements}

The confusion matrices for each of the three participants are shown in Figure 4.A which present the classification performances with post-processing operation. The success rate of the offline classification is shown in Figure 5.A, without (in black) as well as with (in white) post-processing. As can be seen, this state-of-the-art classification architecture is able to determine the voluntary phantom limb movement among a catalog of 12 possible movements, with a success rate varying between $78 \%$ to $94.8 \%$ according to the participant. The performance decreases with $10-15 \%$ when skipping post-filtering operation. This is not surprising since transitions between movements clearly generate an important number of classification errors, which could be due to sEMG labeling approximations and/or to the absence of pause (in this recording protocol for offline classification) in between repetitions by the participants.
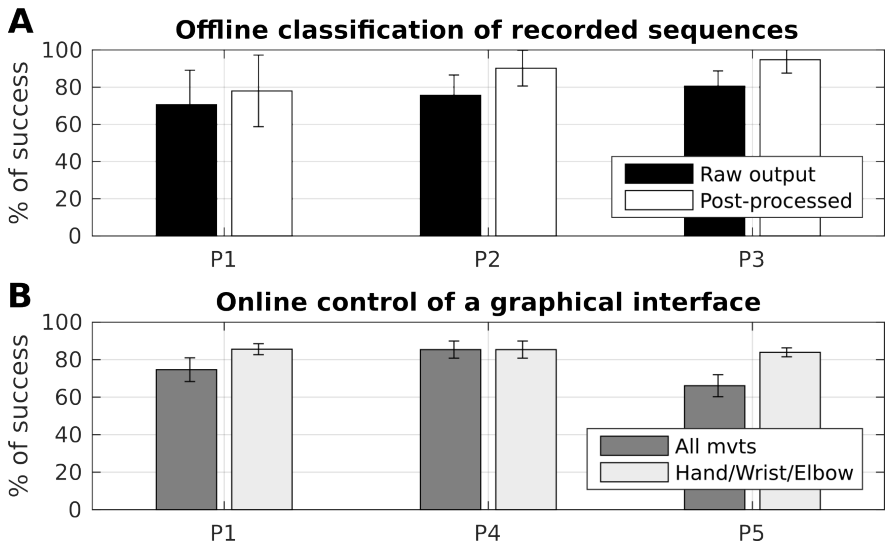

Fig. 5. Plot of the percentages of classification success. A: Success rate (averaged over all movements) obtained during offline classification of prerecorded sequences for each participant, with (white) and without (black) post-processing. B: Success rate obtained during online control of a graphical interface for each participant averaged among all possible movements (6 movements for P4, but 14 for P1 and P5, in dark gray) and averaged among six movements of the three main phantom limb part (hand opening/closing, one wrist movement and elbow flexion/extension, in light grey). 


\section{B. Online control of a graphical user interface}

The confusion matrices for each of the three participants to the online control of graphical interface are shown in Figure 4.B. For the three participants, in addition to their phantom hand and wrist mobilities, the phantom elbow movements (flexion and extension) were added to this session. These phantom movements are less unexpected since the muscles that were naturally associated to these actions (i.e., the biceps and the triceps) are still -at least partially- present in the residual arm. Nonetheless, these movements have been added since having (in addition to finely controlled hand and wrist prosthetics) a natural control over a motorized elbow prosthesis through such a signal would be of great interest for transhumeral amputees. During this control experiment, the participants were asked, after two demonstrations of each of their phantom movement for training of the classifier, to switch on the interface buttons associated to their phantom limb movements. Participants P1 and P5 performed 70 randomized actions (14 movements performed 5 times) and participant 4 , 30 (6 movements performed 5 times). The successful recognition rate averaged over all movements is reduced compared to offline classification of repetitive sequences. Nevertheless, the recognition rate is still high: $74.6 \%$ for $\mathrm{P} 1,85.3 \%$ for $\mathrm{P} 4$ and $66.1 \%$ for $\mathrm{P} 5$. The high success rate of $\mathrm{P} 4$ could be direcly related to the reduced size of his controllable phantom limb movements: indeed, when considering only the performance of P1 and P5 over a reduced set of hand (opening/closing), two wrist action (pronation/supination for P4 or flexion/extension for P5) and elbow (flexion/extension), similar rates are obtained ( $85.5 \%$ for $\mathrm{P} 1$ and $83.9 \%$ for P5), as it can be seen on Figure 5.B). Figure 6 presents the evolution
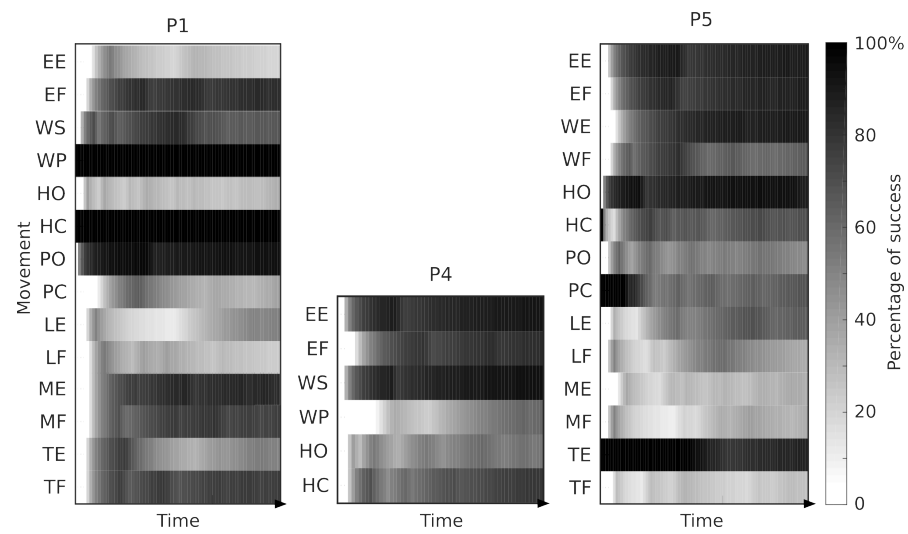

Fig. 6. Online control performance as a function of the time. The color scale increases from white to black as a function of increasing classification rate.

of the performance as a function of time (normalized for all phantom movements since each of them required different time to be performed). It can be seen that some phantom movements are directly and constantly perfectly recognized (HC and WP in P1), while for some others the success rate start to decrease after a certain time (like PC or HO in P5). A few movements seems also to become more consistent across the session time (ME and MF in P1, EF in P4 or WE in P5). Since the control session, after the training phase lasted approximatively one hour, this is a first indicator of the stability of these patterns, at least for a short time period.

\section{Dimensionality of the sEMG patterns}

In order to determine the actual available dimensionality and to evaluate the redundancy among the group of sEMG electrodes, a simple Principal Component Analysis (PCA) [24] was run over the training data sets (transformed into features) of the five participants. Fig. 7 presents the explained cumulative variance as successive numbers of Principal Components (PCs) are used to reconstruct the training data in the sEMG space. It can be seen that, while for offline recognition of sequences, the first 6 PCs explain more than $99.5 \%$ of the data variance, it is necessary to consider more PCs (10 to explain similar variance) for online control based on phantom limb movements (apart from the case of $\mathrm{P} 4$ which has been controlling a reduced set of movements).

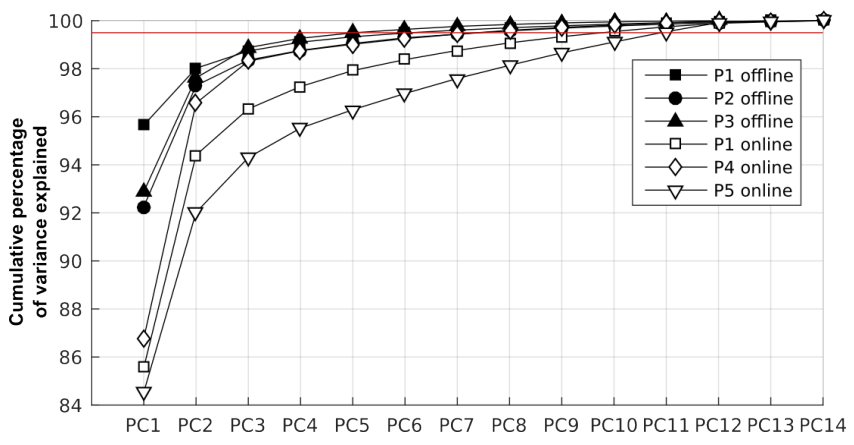

Fig. 7. Plot of the cumulative variance explained as successive numbers of PCs are used to reconstruct the training data. The red horizontal line represents $99.5 \%$ of the explained variance.

\section{Discussion}

In this study we showed that with a state-of-the-art classification architecture (LDA classifier with a set of 4 features) it is possible to classify mobile phantom limb activity with a rather important average success rate (over $80 \%$ when considering basic sets of six hand, wrist and elbow movements). Moreover, we showed that it is possible to use pattern recognition output to give the control of a device (here a graphical interface) to these transhumeral amputees. This is clearly an encouraging result since it is similar to classification performances of surface EMG activity on forearm amputees (80\% in [25]) that are generally obtained with a reduced catalog of movements (4 to 6) of the phantom fingers. Moreover, the present result was achieved with a limited training of the algorithm (two demonstrations of each movements during the training phase) and no specific training of the participants (none of them was used to mobilize their phantom limb).

\section{A. Dimensionality of phantom limb associated sEMG patterns}

The control dimensionality of this phantom limb associated muscle activity seems rather important since three participants 
were able to control 6 different movements with a successful classification rate over $83 \%$. These rates are slightly reduced when including the recognition performance of more fine gestures, like individual fingers movements. Nonetheless, the catalog of gesture when defined including two "multiple fingers actions" (hand and pinch opening and closing) clearly had an impact on the overall performance. Indeed, the confusion matrices of P1 (online) and particularly P5 show an important confusion between whole hand movement and pinch movement, which is not surprizing because of their similarity. Similar phenomenon occured in P2 with the opening of the three fingers that looked like a hand opening. A futur catalog of gestures should maximize the difference between its classes.

Beyond the proximity of some movements of the catalog, the reassuring result is that some of the confusions seem to be directly related to natural synergies (coordinations between joints) similarly to those that can be observed in an healthy limb. The most easy-to-understand confusion is that between some finger extensions (like $M E$ or $L E$ ); indeed, one only has to try to see that an extension of the middle finger naturally entails extensions of other fingers. On the opposite way, some confusions seem related to the natural tendencies, when vigorously flexing one specific finger (or closing the pinch), to extend (almost unvoluntarily) other fingers to prevent from their natural tendency to follow each others. The "checkerboard" aspect of the confusion matrices (particularly visible on P5) indicates that finger flexions are generally confused with other flexions closign actions, while extensions are mixed with other extensions or opening actions. The results shown in Fig. 7 on the dimensionality in the sEMG space, give interesting indications on the dimensionality of these patterns. Since the number of muscles in the upper-arm is reduced with respect to that in the forearm, the results obtained on offline sequences is not surprizing. But the rather high number of PCs (which increased for P1 compared to the offline result) found in active control is interesting since it could be an indicator that there are more independent muscular subvolumes in the residual limbs of subjects with a controllable phantom limb, and therefore that some natural reinnervation process could have occurred, to create these subvolumes. Results obtained in monkey studies are in line with this hypothesis [26], [27].

More analysis should be conducted in order to analyze this phenomenon, and through this, to optimize the number and placement of electrodes (as shown in [28], [29] on the different kind of residual limbs that these transhumeral amputated subjects can have.

\section{B. Using phantom limb associated SEMG patterns as a control method in transhumeral amputees}

These five transhumeral amputees (among whom some rather aged) who never received any Targeted Muscle Reinnervation (TMR) surgery which could have extended their control possibilities [12], were able to control their elbow and some basic hand and wrist functions in a real-time experiment. Obviously, successful online recognition and control rate averaged over all movements $(66.1 \%$ to $85.3 \%)$ is reduced compared to offline classification of repetitive sequences (78\% to $94.8 \%$ ).
This is not surprizing since the task is more complex for both the participant (in terms of concentration in particular) and the classification algorithm (since the randomized sequences are more likely to make the sEMG patterns vary). With an average successfull classification rate around $70 \%$ with a catalog of 14 movements, the control of the interface was sometimes a bit shaky, with undesired blinkings of other buttons due to classification confusions, especially during transitions between each button-task and the small following resting pause. Indeed, when relaxing after having switched on one button, the natural tendency (reported by all the participants) of their phantom limb to come back to an intermediate position (for example for the hand, a posture with fingers slightly flexed) when not voluntarily mobilized, appeared to generate very variable myoelectric patterns confusing the classifier. Nonetheless, these blinkings were clearly reduced when participants performed strong actions (i.e., requiring important activations of their residual limb muscles) like whole hand movements, or the wrist and elbow gestures, as illustrated by the higher success rate obtained for these actions shown on Fig. 5.B.

This success rate still remains slightly lower than the latest classification success of over $90 \%$ reported in the literature [30] obtained with a similar classification architecture on transcubital amputees mobilizing their residual hand and wrist muscles. Several reasons exist for this. First, we recorded from muscle groups that were not related to hand or wrist activity before the amputation. Their activity is probably related to cortical and/or neuromuscular reorganization after the amputation ([31], [26], [27]) and might thus not be as stable as the activity of naturally involved muscle groups. Second, the present task of executing phantom movements was unusual and untrained (participant do not use such phantom movement in their daily living activities), and thus cognitively fatiguing. Fatigue and lack of training could explain some of the variations observed in Fig. 6, such as the decrease of performance after a while, or conversely, the increase due to a learning effect occurring within the session (reinforced by the visual feedback sent by the interface).

In this study, since the online control session lasted one hour and a half, we only evaluated the stability of these patterns within approximatively one hour. To produce more rigorous evidence for the possibility of using these sEMG patterns for a concrete daily use, it would be needed to evaluate the stability of these patterns through more repetitive sessions, with various time lapses (from a few hours to a few days or weeks). Having the patients training themselves to perform in a repeatable manner their phantom limb movements could possibly stabilize the associated sEMG patterns and maximize the interclass distance [32], reduce cognitive fatigue and finally improve the robustness of this control approach, as recenlty shown in [33] with forearm amputees for control with their phantom limb. We do also believe that, possibly with some control methods different from pattern recognition like the ones reviewed in [34] or recent alternatives like [35] and [36], these phantom limb mobilities related myoelectric activities could be used in transhumeral amputees to extend their control abilities over prosthetics and in some cases be a sufficient alternative to surgical procedures. 


\section{CONCLUSION}

This study shows that it seems possible in transhumeral amputees to recognize phantom hand, wrist and elbow actions from the sEMG signals measured on the residual limb. The present study showed this $i$ ) for a rather large catalog of movements including some individual fingers actions that are usually hardly classified, and ii) for patients with a very high amputation level, which means that the sEMG was measured on muscle groups that were not naturally involved in finger, hand and wrist movements. This is clearly a difference with the existing studies on decoding phantom finger sEMG related activities on the forearm of transradial amputees. Beyond classifying offline pre-recorded sequences of phantom limb gestures, we tested if this could be a possible control alternative of a prosthetic, illustrating possibilities of these patients with phantom limb mobilities, and the non-necessity in some particular cases of undergoing surgical muscle reinnervation to increase control possibilities. Nonetheless more realistic experiments have now to be conducted, with possibly more robust control methods to test the possibility of using these signals to perform a functional and efficient control of a prosthetics interacting with an environment. Speaking about a long-term and scientific perspectives, deepening our understanding of the mobile phantom limb phenomenon (cortical reorganization, sprouting effect) could strongly extend possibilities of such approaches and, in addition to changing the way this phenomenon is apprehended by both patients and clinicians, offer transhumeral amputees who have a controllable phantom limb (i.e. 85\% according to [7]) a natural way of controlling complex prostheses (with numerous active joints) without complex training, without control tricks to navigate within a catalog of postures, and potentially without requiring surgical intervention.

\section{ACKNOWLEDGMENT}

The authors warmly thank the patients included in this protocol along with the orthopaedic-fitting team, in particular F. Codemard, K. Georges Y. Corbel and B. Juncker. The study was financially supported by the Région Provence-Alpes-Côte d'Azur (project ExplorAmp, 2012, n ${ }^{\circ}$ 2012-07072), the CNRS (DEFI-SENS, 2013, $\mathrm{n}^{\circ}$ 75114; 2014, $\mathrm{n}^{\circ}$ 40507; PEPS INS2I JCJC MOFACO 2015), the ANR (project PhantoMovCOntrol ANR-15-CE19-0008-02) and the Labex SMART (ANR-11LABX-65) supported by French state funds managed by the ANR within the Investissements d'Avenir programme under reference ANR-11-IDEX-0004-02. These public funding sources had no direct involvement, neither in the study design, in the collection, analysis and interpretation of the data, nor in the writing of this paper.

\section{REFERENCES}

[1] L.G. Cohen, S. Bandinelli, T.W. Findley, and M. Hallett. Motor reorganization after upper limb amputation in man. Brain, 114(1):615$627,1991$.

[2] H. Flor, T. Elbert, S. Knecht, C. Wienbruch, C. Pantev, N. Birbaumer, W. Larbig, and E. Taub. Phantom-limb pain as a perceptual correlate of cortical reorganization following arm amputation. Nature, 1995.
[3] V.S. Ramachandran and W. Hirstein. The perception of phantom limbs. the do hebb lecture. Brain, 121(9):1603-1630, 1998.

[4] WR Henderson and GE Smyth. Phantom limbs. Journal of neurology, neurosurgery, and psychiatry, 11(2):88, 1948.

[5] C.M. Kooijman, P.U. Dijkstra, J.H.B. Geertzen, A. Elzinga, and C.P. van der Schans. Phantom pain and phantom sensations in upper limb amputees: an epidemiological study. Pain, 87(1):33-41, 2000.

[6] J.B. De Graaf, N. Jarrassé, C. Nicol, A. Touillet, T. Coyle, L. Maynard, N. Martinet, and J. Paysant. Phantom hand and wrist movements in upper limb amputees are slow but naturally controlled movements. Neuroscience, 312:48-57, 2016.

[7] A. Touillet, L. Peultier, C. Nicol, N. Jarrassé, I. Loiret, N. Martinet, J Paysant, and JB De Graaf. A detailed description of phantom upper limb movements improves understanding of neuroplasticity after amputation. Annals of Neurology, 2016, submitted.

[8] W. Schady, S. Braune, S. Watson, H.E. Torebjörk, and R. Schmidt. Responsiveness of the somatosensory system after nerve injury and amputation in the human hand. Annals of neurology, 36(1):68-75, 1994.

[9] K.T. Reilly, C. Mercier, M.H. Schieber, and A. Sirigu. Persistent hand motor commands in the amputees' brain. Brain, 129(8):2211-2223, 2006.

[10] C. Mercier, K.T. Reilly, C.D. Vargas, A. Aballea, and A. Sirigu. Mapping phantom movement representations in the motor cortex of amputees. Brain, 129(8):2202-2210, 2006.

[11] M.H. Schieber, C.E. Lang, K.T. Reilly, P. McNulty, and A. Sirigu. Selective activation of human finger muscles after stroke or amputation. In Progress in Motor Control, pages 559-575. Springer, 2009.

[12] T.A. Kuiken, L.A. Miller, R.D Lipschutz, B.A. Lock, K. Stubblefield, P.D. Marasco, P. Zhou, and G.A. Dumanian. Targeted reinnervation for enhanced prosthetic arm function in a woman with a proximal amputation: a case study. The Lancet, 369(9559):371-380, 2007.

[13] M. Huenerfauth and P. Lu. Calibration guide for cyberglove. The City University of New York, Version, 4, 2009.

[14] K. Englehart, B. Hudgins, P.A. Parker, and M. Stevenson. Classification of the myoelectric signal using time-frequency based representations. Medical engineering \& physics, 21(6):431-438, 1999.

[15] J.U. Chu, I. Moon, and M.S. Mun. A real-time emg pattern recognition system based on linear-nonlinear feature projection for a multifunction myoelectric hand. Biomedical Engineering, IEEE Trans. on, 53(11):2232-2239, 2006.

[16] Y. Huang, K.B. Englehart, B. Hudgins, and A.D.C. Chan. A gaussian mixture model based classification scheme for myoelectric control of powered upper limb prostheses. Biomedical Engineering, IEEE Trans. on, 52(11):1801-1811, 2005.

[17] F.H.Y. Chan, Y.S. Yang, F.K. Lam, Y.T. Zhang, and P.A. Parker. Fuzzy emg classification for prosthesis control. Rehabilitation Engineering, IEEE Trans. on, 8(3):305-311, 2000.

[18] A. Phinyomark, F. Quaine, S. Charbonnier, C. Serviere, F. TarpinBernard, and Y. Laurillau. Emg feature evaluation for improving myoelectric pattern recognition robustness. Expert Systems with Applications, 40(12):4832-4840, 2013.

[19] P. Kaufmann, K. Englehart, and M. Platzner. Fluctuating emg signals: Investigating long-term effects of pattern matching algorithms. In Engineering in Medicine and Biology Society (EMBC), Int. Conf of the IEEE, pages 6357-6360, 2010.

[20] M.A. Oskoei and H. Hu. Support vector machine-based classification scheme for myoelectric control applied to upper limb. Biomedical Engineering, IEEE Trans. on, 55(8):1956-1965, 2008.

[21] D. Tkach, H. Huang, and T.A. Kuiken. Research study of stability of time-domain features for electromyographic pattern recognition. $J$ Neuroeng Rehabil, 7:21, 2010.

[22] M. Zardoshti-Kermani, B.C. Wheeler, K. Badie, and R.M. Hashemi. Emg feature evaluation for movement control of upper extremity prostheses. Rehabilitation Engineering, IEEE Trans. on, 3(4):324-333, 1995. 
[23] J.S. Richman and J.R. Moorman. Physiological time-series analysis using approximate entropy and sample entropy. American Journal of Physiology-Heart and Circulatory Physiology, 278(6):H2039-H2049, 2000.

[24] I. Jolliffe. Principal component analysis. John Wiley \& Sons, Ltd,, 2002.

[25] C. Cipriani, C. Antfolk, M. Controzzi, G. Lundborg, B. Rosén, M.C. Carrozza, and F. Sebelius. Online myoelectric control of a dexterous hand prosthesis by transradial amputees. Neural Systems and Rehabilitation Engineering, IEEE Trans. on, 19(3):260-270, 2011.

[26] H.X Qi, Stewart P.W., and J.H. Kaas. Connections of neurons in the lumbar ventral horn of spinal cord are altered after long-standing limb loss in a macaque monkey. Somatosensory \& motor research, 21(34):229-239, 2004.

[27] Carolyn W-H Wu and Jon H Kaas. Spinal cord atrophy and reorganization of motoneuron connections following long-standing limb loss in primates. Neuron, 28(3):967-978, 2000.

[28] D. Staudenmann, I. Kingma, D.F. Stegeman, and J.H. van Dieën. Towards optimal multi-channel emg electrode configurations in muscle force estimation: a high density emg study. Journal of Electromyography and Kinesiology, 15(1):1-11, 2005.

[29] A.J. Young, L.J. Hargrove, and T.A. Kuiken. Improving myoelectric pattern recognition robustness to electrode shift by changing interelectrode distance and electrode configuration. Biomedical Engineering, IEEE Trans. on, 59(3):645-652, 2012.

[30] A.H Al-Timemy, G. Bugmann, J. Escudero, and N. Outram. Classification of finger movements for the dexterous hand prosthesis control with surface electromyography. Biomedical and Health Informatics, IEEE Journal of, 17(3):608-618, 2013.

[31] M. Gagné, S. Hétu, K.T. Reilly, and C. Mercier. The map is not the territory: Motor system reorganization in upper limb amputees. Human brain mapping, 32(4):509-519, 2011.

[32] N.E. Bunderson and T.A. Kuiken. Quantification of feature space changes with experience during electromyogram pattern recognition control. Neural Systems and Rehabilitation Engineering, IEEE Trans. on, 20(3):239-246, 2012.

[33] M.A. Powell, R.R. Kaliki, and N.V. Thakor. User training for pattern recognition-based myoelectric prostheses: improving phantom limb movement consistency and distinguishability. Neural Systems and Rehabilitation Engineering, IEEE Trans. on, 22(3):522-532, 2014.

[34] D. Farina, N. Jiang, H. Rehbaum, A. Holobar, B. Graimann, H. Dietl, and O.C. Aszmann. The extraction of neural information from the surface emg for the control of upper-limb prostheses: emerging avenues and challenges. Neural Systems and Rehabilitation Engineering, IEEE Trans. on, 22(4):797-809, 2014.

[35] M. Ortiz-Catalan, B. Håkansson, and R. Brånemark. Real-time and simultaneous control of artificial limbs based on pattern recognition algorithms. Neural Systems and Rehabilitation Engineering, IEEE Trans. on, 22(4):756-764, 2014.

[36] S. Amsuess, P. Goebel, B. Graimann, and D. Farina. A multi-class proportional myocontrol algorithm for upper limb prosthesis control: validation in real-life scenarios on amputees. Neural Systems and Rehabilitation Engineering, IEEE Trans. on, 23(5):827-836, 2015.

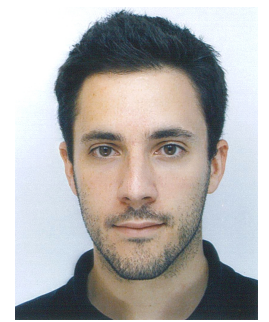

Nathanaël Jarrassé received an M.Sc. in Industrial Systems Engineering from Arts et Mtiers ParisTech, and an M.Sc. and a Ph.D. in Robotics from UPMC, Paris. He has been a postdoctoral Research Associate at the HRG, Department of Bioengineering of Imperial College London, and is now a Tenured Researcher for the National Center for Scientific Research (CNRS) at ISIR. His research focuses on physical human-robot interaction for medical applications, and aims at developing robotic interactive systems (prostheses, exoskeletons, instrumented objects) to study and characterize the human sensorimotor system, and to improve assistance and rehabilitation of individuals affected by motor skill loss.

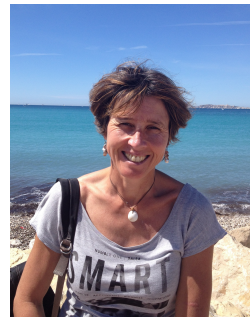

Caroline Nicol performed her PhD under the cosupervision of Pr Paavo V. Komi from the University of Jyväskylä (Finland) and Pr A. Geyssant from the university of St-Etienne (France). She is now an associate professor at the Faculty of Sport Sciences of the Aix-Marseille University. Her research, both fundamental and applied, aims at enriching the understanding of the neuromuscular adjustments to external and prolonged internal disturbances. The present project allows her to study the particularities of the activation patterns associated to phantom arm movements in amputees, their regulation and time evolution with fatigue and with training.

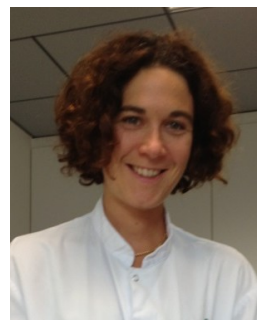

Amlie Touillet is a medical doctor since 2010, specialized in neuro-electrophysiology applied to upper limb pathologies. She actually works at the Louis Pierquin Center in Nancy, France, a rehabilitation institute specialized in orthopedics and traumatology. The medical team work, aiming an optimal compensation of the lost functions, exploits knowledge about central and peripheral neuroplasticity, as well as technologies such as modern prostheses and orthses, in particular with motion capture analysis.

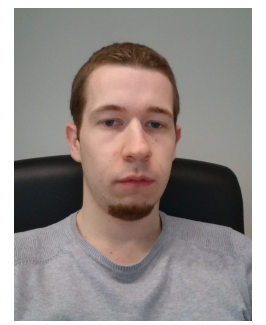

Florian Richer received an M.Eng. degree in Robotics from Polytech'Paris UPMC and a M.Sc. degree in Advanced Systems and Robotics from UPMC, Paris. He is now working as a robotics engineer for the National Center for Scientific Research (CNRS) at the Institute for Intelligent Systems and Robotics (ISIR), Paris, France. 


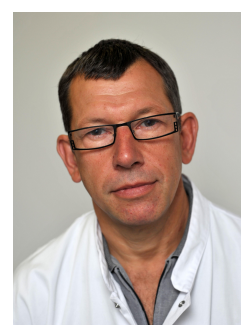

Noel Martinet is Medical Doctor, $\mathrm{PhD}$, specialist in Physical Rehabilitation and Medicine, and diplomed from Biomedical engineering (Metrology) in Lorraine INP. He has been expert in The National Committee for the Evaluation of Medical Devices and Health Technologies (CNEDIMTS) of The French National Authority for Health (HAS).

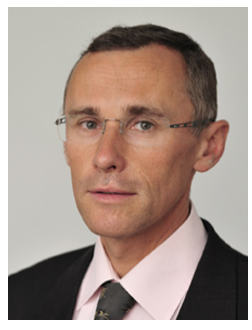

Jean Paysant is Medical Doctor, $\mathrm{PhD}$, Professor of University of Lorraine (France) in Physical Rehabilitation and Medicine, diplomed from Biomechanics and Human Performance in Paris Orsay. His research focuses on neurophysiological integration of movements, particularly in lower limb and upper limb amputees. He is the medical manager of Regional Institute of Rehabilitation (UGECAM NordEst), one of the main PRM Institute in France and well known for Prosthetics and Orthotics Fabrication, Rehabilitation and Clinical Use, Clinical Research for medical devices. He is member of University College of PMR and of European Academy of Rehabilitation Medicine.

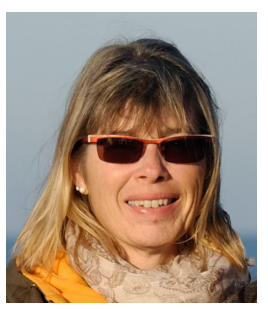

Jozina De Graaf received her M.Sc. in Human Movement Sciences at the Free University in Amsterdam, and her Ph.D. in Behavioral Neuroscience at the University of Technology in Delft, the Netherlands. During post-doctoral training she became an expert in integrative neuroscience and motor control. She is now an associate professor in the Institute of Movement Sciences (ISM) in Marseille, France. Her research focuses on neurophysiological adaptations of the cortical and peripheral sensorimotor system to external (e.g., task requirements) and internal constraints (e.g., lesions and limb amputation). 